\title{
Fungal pathogens and antagonists in root-soil zone in organic and integrated systems of potato production
}

\author{
Leszek Lenc ${ }^{1}$, Hanna Kwaśna²*, Małgorzata Jeske ${ }^{1}$, Krzysztof Jończyk ${ }^{3}$, Czesław Sadowski \\ ${ }^{1}$ Department of Phytopathology and Molecular Mycology, University of Technology and Life Sciences, \\ Kordeckiego 20, 85-225 Bydgoszcz, Poland \\ ${ }^{2}$ Department of Forest Pathology, Poznan University of Life Sciences, Wojska Polskiego 71c, 60-625 Poznań, Poland \\ ${ }^{3}$ Institute of Soil Science and Plant Cultivation (IUNG) State Research Institute, Czartoryskich 8, 24-100 Puławy, Poland
}

Received: January 18, 2016

Accepted: June 3, 2016

\begin{abstract}
Occurrence of culturable Fungi and Oomycota in root-soil habitat of potato cv. Owacja in organic and integrated production systems at Osiny (northern Poland) was compared in 2008-2010. The densities of both pathogens were significantly greater in the organic system. The eudominant fungal taxa (with frequency $>10 \%$ in at least one habitat) included species of Fusarium + Gibberella + Haematonectria, Penicillium, Phoma and Trichoderma. The dominant taxa (with frequency 5-10\%) included species from 13 genera. In the rhizoplane, rhizosphere and non-rhizosphere soil, the total density of potential pathogens was greater in the integrated system, and of potential antagonists in the organic system. Among eudominant and dominant pathogens, Fusarium oxysporum and Gibellulopsis nigrescens occurred at greater density in the integrated system and Haematonectria haematococca and Phoma spp. in the organic system. Among eudominant antagonists, Trichoderma species occurred at greater density in the organic system. The organic system provided more disease suppressive habitat than the integrated system. The occurrence of brown leaf spot and potato blight was however similar in both systems. The mean yield of organic potatoes $\left(24.9 \mathrm{t} \cdot \mathrm{ha}^{-1}\right)$ was higher than the mean organic potato yield in Poland $\left(21.0 \mathrm{t} \cdot \mathrm{ha}^{-1}\right)$ and similar to the mean in other European countries (Germany $25.1 \mathrm{t} \cdot \mathrm{ha}^{-1}$, Great Britain $25.0 \mathrm{t} \cdot \mathrm{ha}^{-1}$ ). The organic system, based on a 5-year rotation, with narrow-leafed lupin, white mustard and buckwheat as a cover crop, inorganic fertilization based on ground rock phosphate + potassium sulphate, and biological and chemical control of insects and diseases (Bacillus thuringiensis ssp. tenebrionis + copper hydroxide + copper oxychloride), may be recommended for use in central Europe.
\end{abstract}

Key words: biological control, cropping system, culturable Fungi and Oomycota, potato

\section{Introduction}

Potato (Solanum tuberosum L.) is the world's most important non-grain food commodity and the fourth main food crop, with $368 \mathrm{mln} \mathrm{t}$ produced in 2013 (Anonymous 2014). The highest production of potatoes has been in eastern and central Europe (about $130 \mathrm{mln} \mathrm{t}$ ) but the most rapid expansion over the past few decades has occurred in southern and eastern Asia (China, India produced about $135 \mathrm{mln} \mathrm{t}$ ). Its status as a cheap and plentiful crop results from its ability to grow in a wide variety of climates and localities. The potato is best known for its carbohydrate (starch) content. It also contains vitamins, minerals and several phytochemicals, such as carotenoids and natural phenols.

Potato can suffer from more than 20 soil-borne diseases (O'Brien and Rich 1979; Fiers et al. 2012; Anonymous 2015). Some can occur on tubers while some on other parts of the plant (Gudmestad et al. 2007) affecting crop growth and usually resulting in yield loss.

Potato soil-borne diseases are the outcome of the compatible interactions between a susceptible host plant, soil-borne pathogen and soil environment. Knowledge of the pathogens as well as factors influencing disease incidence and severity is needed to establish efficient control strategies.

A specific microbial biomass is associated with the potato soil environment. The structure of microbial communities varies with plant age, factors relating to cultivar, nutritional status, and biotic and abiotic stresses (Krechel et al. 2002; Manici and Caputo 2009). About $10^{7}$ bacterial colony-forming units $(\mathrm{cfu}) \cdot \mathrm{g}^{-1}$ of soil live in the potato rhizosphere and potato geocaulosphere (soil around tubers) (Lazarovits et al. 2007). Microbial communities contribute to disease-suppressive effects resulting in lower disease incidence or severity despite the presence of a susceptible host plant, pathogen and favorable climatic conditions (Messiha et al. 2007; Steinberg et al. 2007; Penton et al. 2014). They create two classical types of soil suppressiveness, general and specific suppression, which are a result of, respectively, general activity of the whole microbial biomass, and specific activity of certain individuals or groups of microorganisms (Alabouvette et al. 1996; Weller et al. 2002).

Organic farming relies on agricultural techniques that exclude the use of synthetic chemical pesticides and 
include or recommend organic fertilization. As a result, the plant and soil microbiological environment is quite different from that created by conventional practices and may induce different disease suppression. Changing from a conventional to an organic system involves facing critical disease problems during a transition period of about 5 years, although control of soil-borne diseases can be achieved in the long-term (Bruggen and Termorshuizen 2003). A thorough understanding of how and to what extent crop plants affect the microbiota of soil, and vice versa, is the key to success in both intensive and extensive agricultural systems (Berendsen et al. 2012).

The objective of this study was to compare the fungal communities in potato roots, rhizosphere and non-rhizosphere soil in organic and integrated systems of production. The pathogenic and antagonistic components of the communities are recognized and their significance is estimated. The potential soil suppressiveness in each system is evaluated. The observations were supposed to help in developing strategies for planned procedures used for biological control. This contributes to the general aim of promoting crop management that will improve the soil habitat, increase the soil disease suppression and sustainability and result in greater production profitability.

\section{Materials and Methods}

Potato (S. tuberosum) cv. Owacja was cultivated at Osiny, Poland (52.5371200 N, 21.3282600 E) over three growing seasons (2008-2010). The 8-ha field was divided into two parts; one was under an organic system (4 ha) while another part under integrated system (4 ha). In each system, potato was cultivated on 0.125-ha experimental sub-plots, in rotation. The soil was sandy loam with characteristics presented in Table 1 . The average annual temperature at Osiny was $8.5-9.6^{\circ} \mathrm{C}$ with the highest in $2008 / 2009$ and the average annual precipitation was $537.2-582.8 \mathrm{~mm}$, with the wettest in 2009/2010. Crop management procedures used in the organic and integrated systems of potato production at Osiny in 2008-2010 are presented in Table 2.

Basal roots from 60 randomly chosen plants and 20 samples of non-rhizosphere soil of potato were collect- ed from the A horizon (0-20 cm deep) along a diagonal transect across each of four 0.125-ha sub-plots in each system, at the emergence and flowering stages in 2008-2010.

\section{Isolation of microorganisms}

Sixty apical parts of the basal roots were rinsed in running water for $30 \mathrm{~min}$, then shaken in distilled sterile water $(3 \times 10 \mathrm{~min})$, dried in sterilized blotting paper, cut into 1-cm-long pieces and placed on Potato Dextrose Agar (PDA; 39 g Difco PDA, 11 distilled water, $\mathrm{pH}$ 5.5) in Petri dishes. For isolation of Fungi and Oomycota from the rhizoplane, $10 \mathrm{~g}$ of 1 -cm-long apical parts of the basal roots were rinsed and shaken in a mixture of $90 \mathrm{ml}$ distilled sterile water $+30 \mathrm{~g}$ sterile quartz sand for $10 \mathrm{~min}$. The suspension was serially diluted and $1 \mathrm{ml}$ from each of $10^{-2}$ and $10^{-3}$ dilutions was poured into a Petri dish and covered with liquid $\left(50^{\circ} \mathrm{C}\right)$ Johnson-Martin's agar (JMA; $10 \mathrm{~g}$ glucose, $5 \mathrm{~g}$ peptone, $1 \mathrm{~g} \mathrm{KH}_{2} \mathrm{PO}_{4}, 0.5 \mathrm{~g} \mathrm{MgSO}_{4} \cdot 7 \mathrm{H}_{2} \mathrm{O}$, $0.03 \mathrm{~g}$ rose bengal, $0.0025 \mathrm{~g}$ aureomycin, $20 \mathrm{~g}$ agar, 11 distilled water). For isolation of Fungi and Oomycota from rhizosphere soil, the basal roots of 60 plants were shaken for collection of soil. The soil was mixed and $10 \mathrm{~g}$ was shaken with $90 \mathrm{ml}$ distilled sterile water for $10 \mathrm{~min}$. The suspension was serially diluted and $1 \mathrm{ml}$ from each of $10^{-4}$ and $10^{-5}$ dilutions was poured into a Petri dish and covered with liquid JMA. For isolation of Fungi and Oomycota from non-rhizosphere soil, $1 \mathrm{~g}$ of soil was mixed with $149 \mathrm{~g}$ of sterile quartz sand and $0.02 \mathrm{~g}$ of mixture was put into a Petri dish and covered with liquid JMA. Thirty replicates were used for each dilution and for non-rhizosphere soil. Plates were incubated for $10-30$ days at $25^{\circ} \mathrm{C}$. All microorganisms were identified on the basis of their morphology and sporulation on the specific media (Lenc et al. 2012), according to Pitt (1979), Domsch et al. (1980), Kwaśna et al. (1991) and Seifert et al. (2011).

The density of microorganisms was defined as the number of cfu in a sample. Frequency was defined as the proportion of particular isolates in the total number of isolates. The diversity of a microbial community was defined as the number of species in a sample. A species, or group of related species, was considered as: (i) eudominant

Table 1. Chemical properties of soils at Osiny in 2008-2010

\begin{tabular}{|c|c|c|c|c|c|c|}
\hline \multirow{2}{*}{ Soil characteristics } & \multicolumn{3}{|c|}{ Organic system } & \multicolumn{3}{|c|}{ Integrated system } \\
\hline & 2008 & 2009 & 2010 & 2008 & 2009 & 2010 \\
\hline $\mathrm{pH}$ in $\mathrm{H}_{2} \mathrm{O}$ & 6.32 & 6.53 & 6.41 & 6.38 & 6.43 & 6.76 \\
\hline Humus content [\%] & $1.73 \mathrm{a}$ & 1.54 & $1.63 \mathrm{~b}$ & $1.26 \mathrm{a}$ & 1.44 & $1.32 \mathrm{~b}$ \\
\hline Extractable soil nitrogen & 170.00 abe & 103.00 af & $90.00 \mathrm{bg}$ & 79.00 cde & $61.00 \mathrm{cf}$ & $56.00 \mathrm{dg}$ \\
\hline $\mathrm{NO}_{3}+\mathrm{NH}_{4}\left[\mathrm{mg} \cdot \mathrm{kg}^{-1}\right]^{*}$ & $132.00 \mathrm{abf}$ & $90.00 \mathrm{acg}$ & $51.00 \mathrm{bc}$ & $124.00 \mathrm{~d}$ & $125.00 \mathrm{e}$ & 43.00 defg \\
\hline Extractable soil phosphorus $\left[\mathrm{mg} \cdot \mathrm{kg}^{-1}\right]^{* *}$ & $11.59 \mathrm{ac}$ & $12.87 \mathrm{bd}$ & 9.53 abe & $17.77 \mathrm{c}$ & $15.80 \mathrm{~d}$ & $18.93 \mathrm{e}$ \\
\hline Extractable soil potassium $\left[\mathrm{mg} \cdot \mathrm{kg}^{-1}\right]^{* *}$ & $8.91 \mathrm{ab}$ & $7.80 \mathrm{c}$ & $6.83 \mathrm{ad}$ & $12.43 \mathrm{~b}$ & $14.90 \mathrm{c}$ & $11.67 \mathrm{~d}$ \\
\hline Extractable soil magnesium $\left[\mathrm{mg} \cdot \mathrm{kg}^{-1}\right]^{* * *}$ & $12.49 \mathrm{abc}$ & $9.60 \mathrm{ad}$ & 8.40 be & $7.37 \mathrm{c}$ & $7.33 \mathrm{~d}$ & $12.23 \mathrm{e}$ \\
\hline
\end{tabular}

*analysed with the Kjeldahl method

**analysed with the Egner-Riehm method

***analysed with the Schachtschabel method

a, b, c, d, e, f, g- the same letter in a row shows statistically significant difference between years or systems according to two-way ANOVA (at $p=0.05$ or $p=0.001$ ) 
Table 2. Crop management procedures used in the organic and the integrated systems of potato production at Osiny in 2008-2010

\begin{tabular}{|c|c|c|}
\hline Treatment & Organic system & Integrated system \\
\hline \multirow[t]{5}{*}{ Rotation } & potato & potato \\
\hline & spring wheat & spring wheat \\
\hline & white clover + forage grasses & faba bean \\
\hline & clover + forage grasses & winter wheat \\
\hline & winter wheat & \\
\hline \multirow[t]{3}{*}{ Cover crop } & $\begin{array}{l}\left.2008 \text { - narrow-leafed lupin }\left(120 \mathrm{~kg} \cdot \mathrm{ha}^{-1}\right)\right)+ \text { white } \\
\text { mustard }\left(10 \mathrm{~kg} \cdot \mathrm{ha}^{-1}\right)+\text { buckwheat }\left(20 \mathrm{~kg} \cdot \mathrm{ha}^{-1}\right)\end{array}$ & $\begin{array}{l}2008-\text { white mustard }\left(10 \mathrm{~kg} \cdot \mathrm{ha}^{-1}\right)+\text { buckwheat } \\
\left(40 \mathrm{~kg} \cdot \mathrm{ha}^{-1}\right)\end{array}$ \\
\hline & $\begin{array}{l}2009 \text { - narrow-leafed lupin }\left(70 \mathrm{~kg} \cdot \mathrm{ha}^{-1}\right)+\text { white } \\
\text { mustard }\left(20 \mathrm{~kg} \cdot \mathrm{ha}^{-1}\right)+\text { buckwheat }\left(50 \mathrm{~kg} \cdot \mathrm{ha}^{-1}\right)\end{array}$ & - \\
\hline & $\begin{array}{l}2010 \text { - narrow-leafed lupin }\left(120 \mathrm{~kg} \cdot \mathrm{ha}^{-1}\right)+ \\
\text { buckwheat }\left(40 \mathrm{~kg} \cdot \mathrm{ha}^{-1}\right)\end{array}$ & - \\
\hline \multirow[t]{8}{*}{ Tillage } & \multicolumn{2}{|c|}{2007 - autumn - first plough $(8-10 \mathrm{~cm})$, disc harrowing $(2 \times)$, winter plough $(24-26 \mathrm{~cm})$} \\
\hline & \multicolumn{2}{|c|}{2008 - spring - disc harrowing, cultivation with aggregate Becker, U-1014, rolling, disc harrowing (2×), rolling } \\
\hline & \multicolumn{2}{|c|}{2008 - autumn - post-harvest tillage (gruber), disc harrowing, winter plough $(24-26 \mathrm{~cm})$} \\
\hline & \multicolumn{2}{|c|}{$\begin{array}{l}2009 \text { - spring - disc harrowing, cultivation with aggregate Becker, U-1014, cultivation with aggregate Crone, } \\
\text { Landini, ridging }(2 \times)\end{array}$} \\
\hline & $\begin{array}{l}2009 \text { - autumn - first plough }(8-10 \mathrm{~cm}) \text {, disc } \\
\text { harrowing }(2 \times) \text {, winter plough }(22-24 \mathrm{~cm})\end{array}$ & $\begin{array}{l}2009-\text { autumn - post-harvest tillage (grubber } \\
16-18 \mathrm{~m}), \text { winter plough }(22-24 \mathrm{~cm})\end{array}$ \\
\hline & $\begin{array}{l}2010 \text { - spring - disc harrowing, cultivation with } \\
\text { aggregate Landini, disc harrowing (2-), ridging (2-), } \\
\text { disc harrowing, ridging }\end{array}$ & $\begin{array}{l}2010 \text { - spring - disc harrowing, cultivation with } \\
\text { aggregate Becker, Landini, ridging }(2 \times)\end{array}$ \\
\hline & \multicolumn{2}{|c|}{2007 - autumn - first plough $(8-10 \mathrm{~cm})$, disc harrowing $(2 \times-1 \times)$, winter plough $(24-26 \mathrm{~cm})$} \\
\hline & \multicolumn{2}{|c|}{2008 - spring - disc harrowing, cultivation with aggregate Becker, U-1014, rolling, disc harrowing (2×), rolling } \\
\hline Organic fertilizers & \multicolumn{2}{|l|}{ compost - under potato, in October, $25-30 \mathrm{dt} \cdot \mathrm{ha}^{-1}$} \\
\hline \multirow{4}{*}{$\begin{array}{l}\text { Inorganic } \\
\text { fertilizers applied } \\
\text { late March-early } \\
\text { April }\end{array}$} & ground phosphate rock + potassium sulphate & Polifoska 8 \\
\hline & $2008-72.5 \mathrm{~kg} \mathrm{P}_{2} \mathrm{O}_{5}+62.5 \mathrm{~kg} \mathrm{~K} \mathrm{O}_{2} \cdot \mathrm{ha}^{-1}$ & $2008-22 \mathrm{~kg} \mathrm{~N}+66 \mathrm{~kg} \mathrm{P} \mathrm{O}_{5}+66 \mathrm{~kg} \mathrm{~K}{ }_{2} \mathrm{O} \cdot \mathrm{ha}^{-1}$ \\
\hline & $2009-36.5 \mathrm{~kg} \mathrm{P} \mathrm{O}_{5}+62.5 \mathrm{~kg} \mathrm{~K} 2 \mathrm{O} \cdot \mathrm{ha}^{-1}$ & $2009-15 \mathrm{~kg} \mathrm{NH}{ }_{4}+50 \mathrm{~kg} \mathrm{P} \mathrm{O}_{5}+75 \mathrm{~kg} \mathrm{~K} \mathrm{~K}_{2} \mathrm{O} \cdot \mathrm{ha}^{-1}$ \\
\hline & $2010-29 \mathrm{~kg} \mathrm{P} \mathrm{O}_{5}+62.5 \mathrm{~kg} \mathrm{~K}{ }_{2} \mathrm{O} \cdot \mathrm{ha}^{-1}$ & $2010-20 \mathrm{~kg} \mathrm{NH} H_{4}+60 \mathrm{~kg} \mathrm{P}_{2} \mathrm{O}_{5}+60 \mathrm{~kg} \mathrm{~K} \mathrm{O} \cdot \mathrm{ha}^{-1}$ \\
\hline \multirow[t]{10}{*}{ Pesticides } & Bacillus thuringiensis subsp. tenebrionis (Novodor SCa) & $\begin{array}{l}\text { linuron + clomazon (Afalon Dispersive } 450 \mathrm{SC}^{\mathrm{c}}+ \\
\text { Command } 480 \mathrm{EC}^{\mathrm{c}} \text { ) }\end{array}$ \\
\hline & $2009-7.51 \cdot$ ha $^{-1}$ in 3 doses in June-July & $2008-2010-11+0.11 \cdot$ ha $^{-1}$ in May \\
\hline & \multirow{2}{*}{\multicolumn{2}{|c|}{$2010-131 \cdot$ ha $^{-1}$ in 4 doses in June-July }} \\
\hline & & \\
\hline & Copper hydroxide (Funguran $\mathrm{OH} 50 \mathrm{WP}^{\mathrm{b}}$ ) & $2008-160 \mathrm{~g} \cdot \mathrm{ha}^{-1}$ in two doese in June and July \\
\hline & $2008-2010-2.0 \mathrm{~kg} \mathrm{~h} \cdot \mathrm{ha}^{-1}$ in July & $2009-2010-80 \mathrm{~g} \cdot \mathrm{ha}^{-1}$ in June- July \\
\hline & \multirow{4}{*}{$\begin{array}{l}\text { Copper oxychloride (Miedzian Extra } 350 \mathrm{SC}^{\mathrm{b}} \text { ) } \\
2008-2009-41 \cdot \mathrm{ha}^{-1} \text { in July; } 2010-21 \cdot \mathrm{ha}^{-1} \text { in July }\end{array}$} & mancozeb + metalaxyl (Ridomil Gold MZ 68 WG $^{\mathrm{b}}$ ) \\
\hline & & 2008-2010 $2 \mathrm{~kg} \cdot \mathrm{ha}^{-1}$ in July \\
\hline & & mancozeb + cymoxanil (Curzate M 72,5 WG') \\
\hline & & $2008-20102 \mathrm{~kg} \cdot \mathrm{ha}^{-1}$ in July \\
\hline
\end{tabular}

Removal of weeds harrowing or manually during vegetation

harrowing

Compost included solid cattle manure enriched with grasses and clover and provided $2.8 \mathrm{t} \cdot \mathrm{ha}^{-1}$ of organic matter and $0.1 \mathrm{t} \cdot \mathrm{ha} \mathrm{a}^{-1}$ of $\mathrm{N} ; 0.04 \mathrm{t} \cdot \mathrm{ha}^{-1}$ of P; $0.1 \mathrm{t} \cdot \mathrm{ha}^{-1}$ of $\mathrm{K} ; 0.25 \mathrm{t} \cdot \mathrm{ha}^{-1}$ of Ca; $0.27 \mathrm{t} \cdot \mathrm{ha}^{-1}$ of Mg; $0.005 \mathrm{t} \cdot \mathrm{ha}^{-1}$ of Na. Before planting (end of April), the soil was ploughed and cultivated to create a deep tilth. Tubers were planted in earthed-up ridges.

${ }^{a}$ insecticide; bfungicide; cherbicide 
- with frequency $>10 \%$; (ii) dominant - with frequency 5-10\%; (iii) subdominant - with frequency $2-5 \%$; (iv) recedent - with frequency $1-2 \%$; (v) sub recedent - with frequency $<1 \%$ (Tischler 1949).

\section{Assessment of potato diseases}

The occurrence of brown leaf spot [Alternaria alternata (Fries) Keissler] and potato blight [Phytophthora infestans (Mont.) de Bary] was assessed on 100 plants collected from random sites along a diagonal transect across each of the four replicate 0.125 -ha sub-plots in each system. Potatoes in both systems were given values from 1 to 9 based on increasing proportion of leaf area affected. Potato tuber yield $\left(\mathrm{t} \cdot \mathrm{ha}^{-1}\right)$ was determined for each system.

\section{Statistical analysis}

Chemical properties ( $\mathrm{pH}$, and content of humus, nitrogen, phosphorus, potassium and magnesium) of the soil were compared between two systems (organic and integrated) and three years $(2008,2009$ and 2010) by two-way ANOVA analyses (at $p=0.05$ or $p=0.001$ ). Density and diversity of fungi and potato tuber yield were compared between two systems and three years (2008, 2009 and 2010) by $\chi^{2}$ tests. Relationships between density of fungi and soil chemical properties were estimated by Pearson's correlation coefficient. All analyses were done separately for roots, rhizoplane, rhizosphere soil and non-rhizosphere soil.

\section{Results}

Densities of Fungi + Oomycota in potato roots, rhizoplane, rhizosphere soil and non-rhizosphere soil in individual years were greater (often significantly, $\mathrm{p}=0.05$ or $\mathrm{p}=0.001$ ) in the integrated system (Table 3). Densities of Fungi + Oomycota in rhizoplane, rhizosphere soil and non-rhizosphere (organic system) were usually significantly greater, in 2009 than in 2008 or 2010.

Fungi and Oomycota identified from each system were grouped into dominance classes (Table 4) and, where possible, affiliated according to their known ecological functions, i.e. as potential pathogens or antago- nists. Eudominants and dominants included Zygomycota and Ascomycota fungi. Subdominants, apart from Ascomycota, included also Oomycota represented by Pythium spp. The total frequencies of eudominants + dominants in roots, rhizoplane, and rhizosphere and non-rhizosphere soil were similar in the two systems (77.5-98.6\%).

The total density of potential potato pathogens was greater in the integrated system, significantly $(p=0.05$ or $\mathrm{p}=0.001$ ) in all habitats except roots (Table 5). Densities of Alternaria alternata, Gibberella intricans, Haematonectria haematococca, Phoma spp. and Rhizopus were usually significantly greater in the organic system, and densities of C. coccodes (mostly in rhizoplane and rhizosphere), Cladosporium herbarum, Fusarium culmorum, F. oxysporum, Gibellulopsis nigrescens, Pythium spp. and Stemphylium sp. were greater in the integrated system (Table 4; some taxa not shown separately).

The total density of potential antagonists was significantly greater in the organic system, $(p=0.001)$ for rhizoplane and rhizosphere soil (Table 5). Densities of Mortierellales + Mucorales and Trichoderma spp. were usually significantly greater in the organic system, and densities of Chaetomium spp., Paecilomyces + Purpureocillium and Sarocladium strictum in the integrated system (Table 4 ). Densities of Penicillium spp. were significantly greater in the integrated system.

The ratio of pathogen density to antagonist density was less in the organic system than in the integrated system in all habitats (Table 5).

Acidity of soil was similar in the two systems (Table 1). Humus, nitrogen and magnesium contents were usually significantly higher in the organic system and phosphorus and potassium content in the integrated system (Table 1). In roots, rhizoplane, rhizosphere and non-rhizosphere soil there was no correlation between density of fungi and soil $\mathrm{pH}$. There was negative correlation between mean density of fungi and humus content $(r=-0.8356,-0.6471,-0.5853$, -0.7716 , respectively in roots, rhizoplane, rhizosphere and non-rhizosphere soil; $\mathrm{p}<0.0001)$, nitrogen content $(r=-0.4522,-0.8005,-0.5615,-0.3411 ; \mathrm{p}<0.0001)$ and magnesium content $(r=-0.0704,-0.6121,-0.6752,-0.5878$; $\mathrm{p}<0.0001$ ), and positive correlation between mean density of fungi and phosphorus content $(r=0.7319,0.3653$,

Table 3. Density (no. of colony forming units in a sample) of Fungi and Oomycota in potato cv. Owacja at Osiny in 2008-2010

\begin{tabular}{|c|c|c|c|c|c|}
\hline Habitat & Cropping system & 2008 & 2009 & 2010 & 2008-2010 total \\
\hline \multirow{2}{*}{ Roots } & $\mathrm{O}$ & 88 & 93 & 90 & 271 \\
\hline & I & 104 & 88 & 100 & 292 \\
\hline \multirow{2}{*}{ Rhizoplane } & $\mathrm{O}$ & $110 \mathrm{a}, \mathrm{A}, \mathrm{B}$ & $189 \mathrm{~A}$ & $169 \mathrm{~B}$ & 468 \\
\hline & $\mathrm{I}$ & $181 \mathrm{a}$ & 172 & 171 & 524 \\
\hline \multirow{2}{*}{ Rhizosphere soil } & $\mathrm{O}$ & $93 \mathrm{a}, \mathrm{A}$ & $158 \mathrm{~A}, \mathrm{~B}$ & $109 \mathrm{~B}$ & $360 \mathrm{~b}$ \\
\hline & I & $151 \mathrm{a}, \mathrm{A}$ & $156 \mathrm{~B}$ & 119 A,B & $426 \mathrm{~b}$ \\
\hline \multirow{2}{*}{ Non-rhizosphere soil } & $\mathrm{O}$ & $258 \mathrm{a}, \mathrm{A}$ & $322 \mathrm{~A}, \mathrm{~B}$ & $239 \mathrm{~b}, \mathrm{~B}$ & 819 a \\
\hline & I & $497 \mathrm{a}, \mathrm{A}, \mathrm{B}$ & $327 \mathrm{~A}$ & $302 \mathrm{~b}, \mathrm{~B}$ & 1126 a \\
\hline
\end{tabular}

O - organic system; I - integrated system

$\mathrm{a}, \mathrm{b}$ - the same lower case letter in a column shows statistically significant difference between systems according to $\chi^{2}$ tests at $\mathrm{p}=0.001$ or $\mathrm{p}=0.05$ respectively

$\mathrm{A}, \mathrm{B}$ - the same upper case letter in a row shows statistically significant difference between years according to $\chi^{2}$ tests at $\mathrm{p}=0.001$ or $\mathrm{p}=0.05$ 
Table 4. Frequency (\%) of taxa of fungi and Oomycota in potato cv. Owacja at Osiny in 2008-2010

\begin{tabular}{|c|c|c|c|c|c|}
\hline Taxon or group of taxa & $\begin{array}{l}\text { Production } \\
\text { system }\end{array}$ & In roots & $\begin{array}{l}\text { In } \\
\text { rhizoplane }\end{array}$ & $\begin{array}{l}\text { In } \\
\text { rhizosphere } \\
\text { soil }\end{array}$ & $\begin{array}{l}\text { In non- } \\
\text { rhizosphere } \\
\text { soil }\end{array}$ \\
\hline \multicolumn{6}{|c|}{ Eudominants (frequency $>10 \%$ in at least one habitat) } \\
\hline \multicolumn{6}{|l|}{ Ascomycota } \\
\hline \multirow{2}{*}{$\begin{array}{l}\text { Fusarium culmorum (W. G. Sm.) Sacc. + F. oxysporum } \\
\text { Schlecht. emend. Snyd. et Hans. + F. sporotrichioides Sherb. + } \\
\text { Gibberella avenacea R.J. Cook + G. intricans Wollenw. + } \\
\text { G. tricincta El-Gholl, McRitchie, Schoult. \& Ridings + G. zeae } \\
\text { (Schwein.) Petch + Haematonectria haematococca (Berk. \& } \\
\text { Broome) Samuels \& Rossman }\end{array}$} & $\mathrm{O}$ & $33.7 \mathrm{~b}$ & $29.8 \mathrm{a}$ & 11.8 & $17.0 \mathrm{~b}$ \\
\hline & I & $43.8 \mathrm{~b}$ & $41.8 \mathrm{a}$ & 13.4 & $16.7 \mathrm{~b}$ \\
\hline \multirow[t]{2}{*}{ Including F. oxysporum } & $\mathrm{O}$ & $7.4 \mathrm{a}$ & $6.4 \mathrm{a}$ & $2.2 \mathrm{a}$ & $7.6 \mathrm{a}$ \\
\hline & $\mathrm{I}$ & $31.8 \mathrm{a}$ & $29.0 \mathrm{a}$ & $10.8 \mathrm{a}$ & $11.5 \mathrm{a}$ \\
\hline \multirow[t]{2}{*}{ Including H. haematococca } & $\mathrm{O}$ & $25.5 \mathrm{a}$ & $17.5 \mathrm{a}$ & $5.3 \mathrm{a}$ & $7.4 \mathrm{a}$ \\
\hline & I & $11.0 \mathrm{a}$ & $2.7 \mathrm{a}$ & $1.2 \mathrm{a}$ & $3.6 \mathrm{a}$ \\
\hline \multirow[t]{2}{*}{ Penicillium spp. } & $\mathrm{O}$ & $6.3 \mathrm{a}$ & $21.2 \mathrm{a}$ & $24.7 \mathrm{a}$ & $34.9 \mathrm{a}$ \\
\hline & I & $14.4 \mathrm{a}$ & $33.6 \mathrm{a}$ & $37.3 \mathrm{a}$ & $49.6 \mathrm{a}$ \\
\hline \multirow{2}{*}{$\begin{array}{l}\text { Phoma eupyrena Sacc. + P. glomerata (Corda) Wollenw. \& } \\
\text { Hochapfel }\end{array}$} & $\mathrm{O}$ & 3.0 & 1.7 & 10.3 & 3.9 \\
\hline & $\mathrm{I}$ & 0 & 1.1 & 7.3 & 2.1 \\
\hline \multirow{2}{*}{$\begin{array}{l}\text { Trichoderma hamatum (Bon.) Bain. + T. harzianum Rifai + } \\
\text { T. koningii Oudem. + T. polysporum (Link ex Pers.) Rifai + } \\
\text { T. viride Pers. ex Gray }\end{array}$} & $\mathrm{O}$ & $51.3 \mathrm{~b}$ & $28.0 \mathrm{a}$ & $35.0 \mathrm{a}$ & $26.2 \mathrm{a}$ \\
\hline & I & $33.9 \mathrm{~b}$ & $11.9 \mathrm{a}$ & $6.6 \mathrm{a}$ & 9.7 a \\
\hline \multicolumn{6}{|c|}{ Dominants (frequency $5-10 \%$ in at least one habitat) } \\
\hline \multicolumn{6}{|l|}{ Zygomycota } \\
\hline \multirow{2}{*}{$\begin{array}{l}\text { Absidia glauca Hagem + Actinomucor elegans (Eidam) C.R. Benj. } \\
\text { \& Hesselt. + Mortierella alpina Peyronel + M. exigua Linnem. } \\
+ \text { M. hyalina (Harz) W. Gams + Mucor circinelloides Tiegh. }+ \\
\text { M. moelleri (Vuill.) Lendn. + M. mucedo Fresen + Rhizopus } \\
\text { arrhizus A. Fisch. }\end{array}$} & $\mathrm{O}$ & 0.7 & $5.3 \mathrm{~b}$ & 4.8 & $2.2 \mathrm{a}$ \\
\hline & I & 0.6 & $1.6 \mathrm{~b}$ & 3.3 & $4.3 \mathrm{a}$ \\
\hline \multicolumn{6}{|l|}{ Ascomycota } \\
\hline \multirow{2}{*}{$\begin{array}{l}\text { Clonostachys rosea f. catenulata (J.C. Gilman \& E.V. Abbott) } \\
\text { Schroers + C. rosea f. rosea (Link) Schroers Samuels. Seifert \& } \\
\text { W. Gams + C. solani f. solani (Harting) Schroers \& W. Gams }\end{array}$} & $\mathrm{O}$ & 3.3 & 3.6 & 4.4 & 2.1 \\
\hline & I & 5.9 & 4.0 & 3.7 & 1.9 \\
\hline \multirow{2}{*}{$\begin{array}{l}\text { Gibellulopsis nigrescens (Pethybr.) Zare, W. Gams \& } \\
\text { Summerb. }\end{array}$} & $\mathrm{O}$ & - & - & $1.1 \mathrm{a}$ & 0.9 \\
\hline & I & - & 0.8 & $5.9 \mathrm{a}$ & 2.1 \\
\hline
\end{tabular}

Subdominants (frequency $2-5 \%$ in at least one habitat)

\begin{tabular}{|c|c|c|c|c|c|}
\hline \multicolumn{6}{|l|}{ Ascomycota } \\
\hline \multirow{2}{*}{$\begin{array}{l}\text { Alternaria alternata (Fr.) Keissl. + Cladosporium herbarum } \\
\text { (Pers.) Link + Stemphylium sp. }\end{array}$} & $\mathrm{O}$ & 0.7 & 1.9 & - & 1.3 \\
\hline & I & 0.3 & 2.1 & 0.2 & 1.0 \\
\hline \multirow[t]{2}{*}{ Chaetomium funicola Cooke + Ch. indicum Corda } & $\mathrm{O}$ & - & 0.2 & 0.6 & 3.4 \\
\hline & I & - & 0.4 & 1.4 & 3.8 \\
\hline \multirow{2}{*}{$\begin{array}{l}\text { Gliomastix cerealis (P. Karst.) C.H. Dickinson } \\
+ \text { G. murorum (Corda) S. Hughes }\end{array}$} & $\mathrm{O}$ & - & - & $0.3 \mathrm{a}$ & 1.5 \\
\hline & I & - & 0.4 & $3.1 \mathrm{a}$ & 0.7 \\
\hline \multirow[t]{2}{*}{ Paraconiothyrium fuckelii (Sacc.) Verkley \& Gruyter } & $\mathrm{O}$ & - & 2.8 & 0.3 & 0.1 \\
\hline & I & - & - & 0.5 & 0.2 \\
\hline \multirow{2}{*}{$\begin{array}{l}\text { Paecilomyces niveus Stolk \& Samson + P. variotii Bainier + } \\
\text { Paecilomyces spp.+ Purpureocillium lilacinum (Thom) Luangsa- } \\
\text { ard, Houbraken, Hywel-Jones \& Samson }\end{array}$} & $\mathrm{O}$ & - & 0.2 & $0.6 \mathrm{a}$ & 1.2 \\
\hline & I & - & 1.3 & $4.0 \mathrm{a}$ & 1.8 \\
\hline \multirow[t]{2}{*}{ Sarocladium strictum (W. Gams) Summerb. } & $\mathrm{O}$ & 0.4 & 1.9 & 0.6 & 0.1 \\
\hline & I & 1.0 & 0.2 & 2.1 & 0.9 \\
\hline \multicolumn{6}{|l|}{ Oomycota } \\
\hline \multirow[t]{2}{*}{ Pythium spp. } & $\mathrm{O}$ & - & - & - & - \\
\hline & I & - & - & 2.1 & 0.1 \\
\hline \multicolumn{6}{|c|}{ Recedents (frequency $1-2 \%$ in at least one habitat) } \\
\hline \multirow[t]{2}{*}{ Colletotrichum coccodes (Wallr.) S.J. Hughes } & $\mathrm{O}$ & - & 0.4 & 0.6 & - \\
\hline & I & - & 0.6 & 1.2 & - \\
\hline
\end{tabular}


Table 4. Frequency (\%) of taxa of fungi and Oomycota in potato cv. Owacja at Osiny in 2008-2010 - continuation

\begin{tabular}{lcccccc}
\hline \multicolumn{1}{c}{ Taxon or group of taxa } & $\begin{array}{c}\text { Production } \\
\text { system }\end{array}$ & In roots & $\begin{array}{c}\text { In } \\
\text { rhizoplane }\end{array}$ & $\begin{array}{c}\text { In } \\
\text { rhizosphere } \\
\text { soil }\end{array}$ & $\begin{array}{c}\text { In non- } \\
\text { rhizosphere } \\
\text { soil }\end{array}$ \\
\hline Myrothecium roridum Tode & $\mathrm{O}$ & - & 0.9 & - & - \\
\hline Non-sporulating mycelia & $\mathrm{I}$ & - & 0.2 & 1.2 & - \\
\hline & $\mathrm{O}$ & - & 0.4 & 0.6 & 1.7 \\
\hline Total frequencies of eudominants + dominants & $\mathrm{I}$ & - & - & 0.9 & 1.1 \\
\hline
\end{tabular}

Subrecedents (frequency 0-1\%): Acrostalagmus luteoalbus (Link) Zare, W. Gams \& Schroers, Arthrinium phaeospermum (Corda) M.B. Ellis, Arthrobotrys arthrobotryoides (Berl.) Lindau, Aspergillus fumigatus Fresen., A. niger Tiegh., Aspergillus spp., Cephalotrichum microsporum (Sacc.) P.M. Kirk, Dendryphion nanum (Nees) S. Hughes, Epicoccum nigrum Link, Gonytrichum macrocladum (Sacc.) S. Hughes, Gymnoascus reessii Baran., Humicola grisea Traaen, Leptosphaeria coniothyrium (Fuckel) Sacc., Melanospora spp., Microascus brevicaulis S.P. Abbott, Monographella nivalis (Schaffnit) E. Müll., Papulaspora irregularis Hotson, Pseudogymnoascus pannorum (Link) Minnis \& D.L. Lindner, Septonema sp., Sporothrix spp., Thanatephorus cucumeris (A.B. Frank) Donk, Thysanophora penicillioides (Roum.) W.B. Kendr., Torulomyces indicus (S.B. Saksena) M.H. Hashmi, W.B. Kendr. \& E.B.G. Jones, Trichothecium roseum (Pers.) Link, Umbelopsis vinacea (Dixon-Stew.) Arx

In bold - potato pathogens: A. alternata + Cladosporium + Stemphylium sp., C. coccodes (Heilmann et al. 2006; Anonymous 2015), Fusarium spp. (Secor and Salas 2001; Peters et al. 2008 a, b; Estrada et al. 2010), G. nigrescens (Isaac and Harrison 1968), Phoma spp. (Adams 1983), Penicillium spp. (Rich 1983), Pythium spp. (O’Brien and Rich 1979), Rhizopus spp. (Clark and Moyer 1988). Pathogens antagonists include: Chaetomium spp., Mortierella spp., P. pannorum (Tagawa et al. 2010), Coniothyrium spp. (Cheng et al. 2003). S. strictum (Rivera-Varas et al. 2007), P. lilacinum (Jacobs et al. 2003).

Stimulants of plant growth include G. murorum (Khan et al. 2009).

Nematophagous fungi with potential for controlling potato cyst nematodes include Paecilomyces + Purpureocillium (Jacobs et al. 2003). $\mathrm{O}$ - organic system; I - integrated system

$\mathrm{a}, \mathrm{b}$ - the same letter in a column shows statistically significant difference between systems according to $\chi^{2}$ tests at $\mathrm{p}=0.001$ or $\mathrm{p}=0.05$ respectively

Table 5. Density of potential pathogens and antagonists in potato cv. Owacja at Osiny in 2008-2010

\begin{tabular}{|c|c|c|c|c|c|}
\hline Category & $\begin{array}{l}\text { Cropping } \\
\text { system }\end{array}$ & Roots & Rhizoplane & $\begin{array}{l}\text { Rhizosphere } \\
\text { soil }\end{array}$ & $\begin{array}{l}\text { Non- } \\
\text { rhizosphere } \\
\text { soil }\end{array}$ \\
\hline \multirow{2}{*}{ Density of pathogens (no. of cfu in a sample) } & $\mathrm{O}$ & 101 & $158 \mathrm{a}$ & $86 \mathrm{~b}$ & $189 \mathrm{~b}$ \\
\hline & I & 129 & $248 \mathrm{a}$ & $128 \mathrm{~b}$ & $248 \mathrm{~b}$ \\
\hline \multirow{2}{*}{ Total frequency of pathogens [\%] } & $\mathrm{O}$ & 37.4 & 33.8 & 23.8 & 23.1 \\
\hline & I & 44.1 & 47.3 & 30.1 & 22.0 \\
\hline \multirow{2}{*}{ Diversity of pathogens (no. of species) } & $\mathrm{O}$ & 8 & 12 & 14 & 9 \\
\hline & I & 5 & 12 & 12 & 14 \\
\hline \multirow{2}{*}{ Density of antagonists (no. of cfu in a sample) } & $\mathrm{O}$ & 151 & $184 \mathrm{a}$ & $166 \mathrm{a}$ & 288 \\
\hline & I & 121 & $102 \mathrm{a}$ & $90 \mathrm{a}$ & 252 \\
\hline \multirow{2}{*}{ Total frequency of antagonists [\%] } & $\mathrm{O}$ & 55.7 & 39.2 & 46.0 & 35.2 \\
\hline & $\mathrm{I}$ & 41.4 & 19.4 & 21.1 & 22.4 \\
\hline \multirow{2}{*}{ Diversity of antagonists (no. of species) } & $\mathrm{O}$ & 5 & 11 & 14 & 16 \\
\hline & I & 8 & 12 & 11 & 18 \\
\hline \multirow{2}{*}{ Ratio of pathogen: antagonist density } & $\mathrm{O}$ & 0.7 & 0.9 & 0.5 & 0.7 \\
\hline & $\mathrm{I}$ & 1.1 & 2.4 & 1.4 & 1.0 \\
\hline \multirow{2}{*}{$\begin{array}{l}\text { Ratio of pathogen: antagonist density in cv. } \\
\text { Drop (Lenc et al. 2012) }\end{array}$} & $\mathrm{O}$ & 8.9 & 6.0 & 1.9 & 1.5 \\
\hline & I & 9.3 & 0.9 & 1.3 & 1.1 \\
\hline
\end{tabular}

$\mathrm{O}$ - organic system; I - integrated system

$\mathrm{a}, \mathrm{b}$ - the same letter in a column shows statistically significant difference between systems according to $\chi^{2}$ tests at $\mathrm{p}=0.001$ or $\mathrm{p}=0.05$ respectively 
Table 6. Incidence of diseases and yield of potato cv. Owacja at Osiny in 2008-2010

\begin{tabular}{|c|c|c|c|c|c|}
\hline \multirow{2}{*}{ Fungus } & \multicolumn{4}{|c|}{ Level of resistance to } & \multirow{2}{*}{ Mean 2008-2010 } \\
\hline & cropping system & 2008 & 2009 & 2010 & \\
\hline \multirow{2}{*}{$\begin{array}{l}\text { Alternaria alternata } \\
\text { (brown leaf spot) }\end{array}$} & $\mathrm{O}$ & 7.0 & 8.5 & 7.5 & 7.7 \\
\hline & I & 7.5 & 7.5 & 8.0 & 7.7 \\
\hline \multirow{2}{*}{$\begin{array}{l}\text { Phytophthora infestans } \\
\text { (potato late blight) }\end{array}$} & $\mathrm{O}$ & 7.5 & 7.0 & 7.5 & 7.3 \\
\hline & I & 8.0 & 8.5 & 8.0 & 8.2 \\
\hline \multirow[t]{2}{*}{ Yield of potato tubers $\left[\mathrm{t} \cdot \mathrm{ha}^{-1}\right]$} & $\mathrm{O}$ & $26.7 \mathrm{a}$ & $26.0 \mathrm{a}$ & $22.1 \mathrm{a}$ & $24.9 \mathrm{a}$ \\
\hline & I & $46.6 \mathrm{a}, \mathrm{A}$ & $47.6 \mathrm{a}, \mathrm{B}$ & $25.9 \mathrm{~b}, \mathrm{~A}, \mathrm{~B}$ & $40.0 \mathrm{a}$ \\
\hline
\end{tabular}

$\mathrm{O}$ - organic system; I - integrated system

$\mathrm{a}, \mathrm{b}$ - the same letter in a column shows statistically significant difference between systems according to $\chi^{2}$ tests at $\mathrm{p}=0.05$

A, B - the same letter in a row shows statistically significant difference between years according to $\chi^{2}$ tests at $\mathrm{p}=0.05$

$0.4332,0.6459 ; \mathrm{p}<0.0001)$. In rhizosphere and non-rhizosphere soil there was moderate positive correlation between mean density of fungi and potassium content $(r=0.4747, r=0.5253 ; \mathrm{p}<0.0001)$.

Level of resistance to brown leaf spot and potato blight, based on disease evaluation, was 7.0-8.5 (Table 6). The mean level of resistance to brown leaf spot was similar in the two systems. The mean level of resistance to potato blight was higher in the integrated system. Yield in the organic system $\left(22.1-26.7 \mathrm{t} \cdot \mathrm{ha}^{-1}\right)$ was similar in all three years and lower than in the integrated system (not significantly in 2010). Yield in the integrated system was significantly lower in 2010 than in 2008 and 2009.

\section{Discussion}

Potatoes are generally an undemanding vegetable crop to grow, but they are not very easy using organic growing practices. They are susceptible to a few insect pests and diseases that can be a challenge for organic management. They also require relatively high levels of available nutrients in order to obtain good yields.

The exact mechanisms of natural and spontaneous control of root disease in organic or integrated farming are not known. It is generally assumed, however, that organic amendments suppress root diseases by: (i) increasing the general level of microbial activity, resulting in increased competition and/or antagonism in the rhizosphere and creating general and specific suppression; (ii) stimulation of plants by vesicular-arbuscular mycorrizha (VAM) colonization of roots. Measuring microbial biomass and diversity (community structure) has been recommended as a biological indicator of soil quality and soil suppressiveness (Kennedy and Smith 1995; Yao et al. 2000) and has been employed in national and international monitoring programmes.

Organic farming has been shown to stimulate the growth and development of large and diverse soil microbial communities with potential for efficient nutrient cycling and disease suppression (Fraser et al. 1988; Mäder et al. 1995; Yeates et al. 1997; Gunapala and Scow 1998; Abawi and Widmer 2000; Shannon et al. 2002; Sadowski et al. 2002, 2003; Lenc 2006, 2009; Lenc et al. 2011). The increase in microbial biomass can be 10-26\% (Fraser et al. 1994). How- ever, the organic system described here resulted in smaller densities of Oomycota and fungi in potato roots/soil (but particularly in soil) compared with the integrated system. These results agree with earlier studies of Lenc et al. (2012) carried out earlier in the same location (Osiny), in 2005-2007, but on a different potato cultivar (cv. Drop). As previously reported (Lenc et al. 2012), a lower density of fungi was often associated with non-significantly higher soil acidity and higher amounts of humus, nitrogen and magnesium, but not of phosphorus or potassium. Results of earlier studies on effects of soil acidity on the growth of fungi are contradictory. Yamanaka (2003) reported that many saprotrophic species grew well at $\mathrm{pH} 7$, while Blagodatskaya and Anderson (1998) and Bååth and Anderson (2003) found that soil $\mathrm{pH}$ could have either positive or negative effects on densities of fungi and bacteria.

The negative correlation between density of fungi and content of humus, nitrogen and magnesium observed agrees with some other studies in which fungal biomass was found to decrease as a consequence of application of organic and inorganic fertilization (Bardgett et al. 1999; Bloem and Vos 2004; Bittman et al. 2005; de Vries et al. 2006). However, a positive correlation between density of fungi and content of organic matter has also been reported (Alexander 1977; Henriksen and Breland 1999; Vinten et al. 2002). Differences result from the type of studied habitat. The microbiological response to organic matter depends on its $C: N$ ratio. An increased $C: N$ ratio stimulates, while decreased $C: N$ radio inhibits fungal growth, which is affected by accumulation of nitrogen. Organic carbon (C) in soil is transformed, stored and respired by microorganisms. Increased $\mathrm{N}$ content, leading to decreased $\mathrm{C}: \mathrm{N}$ ratio, decreases microbial respiration, which is followed by decrease in microbial biomass (Spohn 2015).

The cover crop applied in Osiny included mostly narrow-leafed lupin, which, like other legumes, fixes nitrogen in a symbiotic interaction with different bacteria in its rhizosphere. This provision of nitrogen decreased the $\mathrm{C}: \mathrm{N}$ ratio.

The humus content greater in the organic system than in the integrated system resulted from the type of cultivated cover crop. The succulent leaves of the annual legume (narrow-leafed lupin), white mustard and buckwheat break down quickly and release nitrogen and other 
nutrients, but the tough and fibrous stems and roots, particularly of narrow-leafed lupin, after repeated cultivation (in 2008, 2009 and 2010), contributed to humus accumulation.

The inhibitory effect of magnesium and stimulatory effect of potassium on growth of fungi, suggested by the present results, has been reported previously (Jones and Jennings 1964).

White mustard used as a cover crop in the organic system may inhibit growth of fungi, including pathogenic F. oxysporum. It has been suggested that fungal suppression results from glucosinolate degradation into biologically active sulphur-containing thiocyanates (Porter 1995; Gardiner et al. 1999). White mustard suppressed potato soil-borne pathogens (Fusarium, Rhizoctonia and Verticillium species) in studies of Collins et al. (2006).

Evaluation of soil suppressiveness in each system was attempted by affiliating most microorganisms according to their known ecological functions. Potential pathogens and antagonists of potato pathogens were quantified.

Potato pathogens included A. alternata (brown spot and black pit), C. coccodes (black dot), Fusarium spp. (Fusarium dry rot and Fusarium wilt), G. nigrescens (Verticillium wilt), Penicillium spp. (blue and green mould rots), Phoma spp. (gangrene) and Pythium spp. (leak).

Fusarium oxysporum and $H$. haematococca were the most frequent identified species. These results obtained from plants during the vegetation differ from density of fungi on dry-rotting tubers, where F. avenaceum, F. coeruleum (Sacc.) Booth, F. culmorum, F. oxysporum, F. sambucinum Fuckel, F. sulphureum Schltdl. were found, with F. sulphureum and F. coeruleum being the most common and considered the most important (WojciechowskaKot and Kiszczak 1981; Kurzawińska and Klima 1999; Kurzawińska and Gajda 2002). However, F. oxysporum was found to be the most frequent pathogen in tubers in Michigan, USA (Gachango et al. 2012).

In addition to Fusarium spp., A. alternata and C. coccodes, with frequencies of $24-28.8 \%$ and $4-6.6 \%$ respectively, have been reported as the most common dominants on tubers with dry rot symptoms (Kurzawińska and Gajda 2002). These species occurred with low frequencies in the present study and were among the subdominants and recedents, respectively. The frequency of $C$. coccodes was greater in the integrated system.

The rhizosphere region in the two systems was also inhabited by beneficial microorganisms known to be effective in biological control (Curl and Truelove 1986). Clonostachys, Trichoderma and Purpureocillium lilacinum were the most widespread. By means of competition, antibiosis and mycoparasitism they are able to decrease the severity of black dot, stem canker, black scurf (Rhizoctonia solani Kuhn.) and Verticillium wilt (Verticillium dahliae Kleb.), as well as nematodes (Davide and Zorilla 1983, 1985; Schirmbock et al. 1994; Kubicek et al. 2001; Howell 2003; Jacobs et al. 2003; Harman et al. 2004 and Fiers et al. 2012). Another fungus such as, Gliomastix murorum, produces gibberellin and promotes plant growth (Khan et al. 2009). The beneficial effects of indigenous biological control have been observed after only four years of organic farming (van Bruggen 1995).
The potential fungal antagonists were among the eudominants, dominants and subdominants, and their high density seems to be essential for soil suppressiveness. Fungal recedents and subrecedents (with individual frequency $<2 \%$ ) were represented by $0.1-9.1 \%$ of species, with the largest population in the soil. They seem to be unimportant, making no contribution to the major biological interrelationships. They may, however, participate in natural and spontaneous control of diseases through optimization of nutrient cycles, particularly during environmental disturbances.

The role of some of the detected fungi is very complex. A few usually considered to be pathogenic (Fusarium or Penicillium species) may be antagonistic to Streptomyces spp. (potato scab) (Tagawa et al. 2010) or parasitic on nematodes (Martinez-Beringola et al. 2013) and hence contribute to soil suppressiveness.

The organic system was more suppressive to potential soil-borne pathogens than the integrated system. It supported less growth of C. coccodes, F. culmorum, F. oxysporum, G. nigrescens and Pythium, but not Alternaria, H. haematococca, Phoma or Rhizopus, and stimulated the growth of Trichoderma but not of other potential antagonists, Mortierella, Paecilomyces or S. strictum. The results are partly, from the specific viewpoint of stimulation and antagonism, in agreement with an earlier study, using a different potato cultivar, in which the integrated system was found to be generally more suppressive to potential soil-borne pathogens (Lenc et al. 2012).

Despite the overall greater suppressiveness of the organic system to soil-borne pathogens, A. alternata was found at similar (low) frequency in the organic and integrated systems and brown leaf spot (A. alternata) and late blight (Ph. infestans) occurred at similar frequencies in the two systems. Fungicides based on copper (Funguran $\mathrm{OH}$ 50 WP, Miedzian Extra 350 SC), used in the organic system to control late blight with a dose $6 \mathrm{~kg} \cdot \mathrm{ha}^{-1}$ per year (as regulated by EU), seem to be no less effective than other, synthetic fungicides used in the integrated system.

The mean yield of potatoes grown in the organic system at Osiny $\left(24.9 \mathrm{t} \cdot \mathrm{ha}^{-1}\right)$ was greater than the average yield of organically grown potatoes in Poland $\left(21.0 \mathrm{t} \cdot \mathrm{ha}^{-1}\right)$ and similar to those in other European countries (Germany, $25.1 \mathrm{t} \cdot \mathrm{ha}^{-1}$, Great Britain, $25.0 \mathrm{t} \cdot \mathrm{ha}^{-1}$, Anonymous 2007). The mean yield per hectare for organically grown potatoes was $62.3 \%$ of the yield achieved in the integrated system. In other European countries, potatoes grown in organic systems achieved only $46 \%$ of the yield in conventional systems (Anonymous 2007). It may be assumed, because of the lower frequency of pathogens, higher frequency of antagonists, absence of disease differences that would affect yield, and acceptable tuber yield, that effective disease suppression was operating in the organic system.

Ratios of pathogen to antagonist densities were consistently smaller in the organic system than in the integrated system. The differences were small in roots and non-rhizosphere soil but more distinct in rhizoplane and rhizosphere soil. In a similar study (Lenc et al. 2012), the pathogen : antagonist density ratios in roots and rhizoplane were much greater (6.0-9.3; Table 5), partly as a result of the high frequency of $C$. coccodes in roots. The ratios were similar in 
rhizosphere and non-rhizosphere soils (1.1-1.9). The higher pathogen : antagonist ratio resulted in much lower crop which on average was only $10.1-15.0 \mathrm{t} \cdot \mathrm{ha}^{-1}$ (Lenc et al. 2012). Since the inconsistencies between the two studies occurred in roots and rhizoplane, it seems likely that the differences were associated with plant lines and their genetic traits (particularly different susceptibilities to C. coccodes). However, the different results may have resulted from differences in management practices (especially if those practices affected incidence of $C$. coccodes).

Effects of plant genotype on the rhizosphere microbiota have been reported previously (Overbeek and van Elsas 2008; Weinert et al. 2009; İnceoğlu et al. 2010). Although often difficult to measure, it is plausible that these differences result from differences between plant cultivar (genotype) in their root-released products (İnceoğlu et al. 2011, 2013).

Comparison of the present results with those of Lenz et al. (2012) raises the major question 'to what extent individual cultivars affect the rhizosphere microbiota'. The answers are of particular significance for development, use and current admission procedures of new cultivars and genetically modified plants (Bruinsma and van Veen 2003) and would define a theoretical area of 'normality' for soil used for potato cropping. Such 'normality' indicated by a 'normal operating range' (NOR) could, according to İnceoğlu et al. (2013), be used as a standard reference for comparison of new cultivars and lines.

The classical method of profiling microbial communities based on pure culture isolation and morphotyping was used because of the scale of the experiment and the limitations of molecular methods. These limitations concern incomplete cell lysis, DNA sorption to soil surfaces, extraction of humic contaminants and inhibitors and DNA degradation (Fatima et al. 2014). Only culture-based techniques allow discrimination between viable members of a community (essential for the evaluation of pathogenantagonist relationships) and inactive (dead) ones. While the possibility that culture-based techniques may mask the presence of slow-growing and non-culturable species must be considered, it may be assumed that the culturable microbial community is representative of the soil habitat complex (including taxa that can not be cultured) and can be considered valid for comparison of two environments.

\section{Conclusions}

The organic system examined for potato cultivation (5year rotation, cover crop of narrow-leafed lupin + white mustard + buckwheat, inorganic fertilization based on ground rock phosphate + potassium sulphate, and biological and chemical control of insects and diseases based on Bacillus thuringiensis subsp. tenebrionis + copper hydroxide + copper oxychloride) resulted in a more diseasesuppressive root/rhizosphere microbiota and similar incidence of leaf disease to the integrated system. The organic system also produced acceptable tuber yields. This system may therefore be suitable for recommendation for organic potato production in central Europe.

\section{Acknowledgements}

The author would like to thank prof. Jan Kuś from the Institute of Soil Science and Plant Cultivation, the State Research Institute in Puławy, and dr Wojciech Nowacki from the Plant Breeding and Acclimatization Institute, National Research Institute, Research Division at Jadwisin, Poland for their cooperation, and for allowing this research.

\section{References}

Abawi G.S., Widmer T.L. 2000. Impact of soil health management practices on soilborne pathogens, nematodes and root diseases of vegetable crops. Applied Soil Ecology 15 (1): 37-47.

Alabouvette C., Hoeper H., Lemanceau P., Steinberg C. 1996. Soil suppressiveness to diseases induced by soilborne plant pathogens. p. 371-413. In: "Soil Biochemistry". Vol. 9 (G. Stotzky, J.M. Bollag, eds.). Marcell Dekker, New York, USA, $568 \mathrm{pp}$.

Alexander M. 1977. Introduction to Soil Microbiology. Wiley, New York, 467 pp.

Anonymous 2007. Potatoes - conventional, IPM and organic systems in Europe. Pesticides News 75: 18-22.

Anonymous 2014. FAOSTAT (Food and Agriculture Organization of the United Nations). Available on: faostat.fao.org [Accessed: May 20, 2015]

Anonymous 2015. List of potato diseases. Available on: http://en.wikipedia.org/wiki/List_of_potato_diseases [Accessed: September 3, 2015]

Bååth E., Anderson T.-H. 2003. Comparison of soil fungal/bacterial ratios in a $\mathrm{pH}$ gradient using physiological and PLFAbased techniques. Soil Biology and Biochemistry 35 (7): 955-963.

Bardgett R.D., Mawdsley J.L., Edwards S., Hobbs P.J., Rodwell J.S., Davies W.J. 1999. Plant species and nitrogen effects on soil biological properties of temperate upland grasslands. Functional Ecology 13 (5): 650-660.

Berendsen R.L., Pieterse C.M.J., Bakker P.A.H.M. 2012. The rhizosphere microbiome and plant health. Trends in Plant Science 17 (8): 478-486.

Bittman S., Forge T.A., Kowalenko C.G. 2005. Responses of the bacterial and fungal biomass in a grassland soil to multiyear applications of dairy manure slurry and fertilizer. Soil Biology and Biochemistry 37 (4): 613-623.

Blagodatskaya E.V., Anderson T.H. 1998. Interactive effects of $\mathrm{pH}$ and substrate quality on the fungal-to-bacterial ratio and $\mathrm{qCO}_{2}$ of microbial communities in forest soils. Soil Biology and Biochemistry 30 (10-11): 1269-1274.

Bloem J., Vos A. 2004. Fluorescent staining of microbes for total direct counts. p. 861-874. In: “Molecular Microbial Ecology Manual" (G. Kowalchuk, F. de Bruijn, I.M. Head, A.J. van der Zijpp, J.D. van Elsas, eds.). Kluwer Academic Publishers, Dordrecht, Netherlands, 853-1044 pp.

Bruggen A.H.C. van 1995. Plant disease severity in high-input compared to reduced-input and organic farming systems. Plant Disease 79 (10): 976-984.

Bruggen A.H.C., Termorshuizen A.J. 2003. Integrated approaches to root disease management in organic farming systems. Australasian Plant Pathology 32 (2): 141-156. 
Bruinsma M., Kowalczuk G.A., Veen J.A. van. 2003. Effects of genetically modified plants on microbial communities and processes in soil. Biology and Fertility of Soils 37: 329-337.

Collins H.P., Alva A., Boydston R.A., Cochran R.L., Hamm P.B., McGuire A., Riga E. 2006. Soil microbial, fungal, and nematode responses to soil fumigation and cover crops under potato production. Biology and Fertility of Soils 42 (3): 247-257.

Curl E.A., Truelove B. 1986. The Rhizosphere. Springer-Verlag, New York, USA, 288 pp.

Davide R.G., Zorilla R.A. 1983. Biological and chemical control studies of the potato cyst nematodes found in Madaymen, Buguias, Benguet. Philippine Phytopathology 19: 28-35.

Davide R.G., Zorilla R.A. 1985. Evaluation of a fungus Paecilomyces lilacinus for the biological control of root-knot nematode Meloidogyne incognita on okra as compared with the nematicide Isazofos. Philippine Agriculturist 68: 493-500.

Domsch K.H., Gams W., Anderson T. 1980. Compendium of Soil Fungi. Academic Press, London-New York-Toronto-SydneySan Francisco, 892 pp.

Fatima F., Pathak N., Verma S.R. 2014. An improved method for soil DNA extraction to study the microbial assortment within rhizospheric region. Molecular Biology International. 6 pp. Available on: http://dx.doi.org/10.1155/2014/518960 [Accessed: May 20, 2015]

Fiers M., Edel-Hermann V., Chatot C., Le Hingrat Y., Alabouvette C., Steinberg C. 2012. Potato soil-borne diseases. A review. Agronomy for Sustainable Development 32 (1): 93-132.

Fraser D.G., Doran J.W., Sahs W.W., Lesoing G.W. 1988. Soil microbial populations and activities under conventional and organic management. Journal of Environmental Quality 17 (4): 585-590.

Fraser P.M., Haynes R.J., Williams P.H. 1994. Effects of pasture improvement and intensive cultivation on microbial biomass, enzyme activities and composition and size of earthworm population. Biology and Fertility of Soils 17 (3): 185-190

Gachango E., Hanson L.E., Rojas A., Hao J.J., Kirk W.W. 2012. Fusarium spp. causing dry rot of seed potato tubers in Michigan and their sensitivity to fungicides. Plant Disease 96 (12): 1767-1774.

Gardiner J.B., Morra M.J., Eberlein C.V., Brown P.D., Borek V. 1999. Allelochemicals released in soil following incorporation of rapeseed (Brassica napus) green manures. Journal of Agriculture and Food Chemistry 47 (9): 3837-3842.

Gudmestad N.C., Taylor R.J., Pasche J.S. 2007. Management of soilborne diseases of potato. Australasian Plant Pathology 36 (2): 109-115.

Gunapala N., Scow K.M. 1998. Dynamics of soil microbial biomass and activity in conventional and organic farming systems. Soil Biology and Biochemistry 30 (6): 805-816.

Harman G.E., Howell C.R., Viterbo A., Chet I., Lorito M. 2004. Trichoderma species - opportunistic, avirulent plant symbionts. Nature Reviews Microbiology 2 (1): 43-56.

Henriksen T.M., Breland T.A. 1999. Nitrogen availability effects on carbon mineralization, fungal and bacterial growth, and enzyme activities during decomposition of wheat straw in soil. Soil Biology and Biochemistry 31 (8): 1121-1134.

Howell C.R. 2003. Mechanisms employed by Trichoderma species in the biological control of plant diseases: the history and evolution of current concepts. Plant Disease 87 (1): 4-10.
İnceoğlu Ö., Al-Soud W.A., Salles J.F., Semenov A.V., Elsas J.D. van. 2011. Comparative analysis of bacterial communities in a potato field as determined by pyrosequencing. PLoS ONE 6 (8): e23321.

İnceoğlu Ö., Salles J.F., Overbeek L.S. van, Elsas J.D. van. 2010. Effects of plant genotype and growth stage on the betaproteobacterial communities associated with different potato cultivars in two fields. Applied and Environmental Microbiology 76 (11): 3675-3684.

İnceoğlu Ö., Overbeek L.S. van, Falcão Salles J., Elsas J.D. van 2013. Normal operating range of bacterial communities in soil used for potato cropping. Applied and Environmental Microbiology 79 (4): 1160-1170. DOI: 10.1128/AEM.02811-12

Jacobs H., Gray S.N., Crump D.H. 2003. Interactions between nematophagous fungi and consequences for their potential as biological agents for the control of potato cyst nematodes. Mycological Research 107 (1): 47-56.

Jones E.B.G., Jennings D.H. 1965. The effect of cations on the growth of fungi. New Phytologist 64 (1): 86-100.

Kennedy A.C., Smith K.L. 1995. Soil microbial diversity and the sustainability of agricultural soils. Plant and Soil 170 (1): 75-86.

Khan S.A., Hamayun M., Kim H.-Y., Lee I.-J., Kim J.-G. 2009. Gibberellin production and plant growth promotion by a newly isolated strain of Gliomastix murorum. World Journal of Microbiology and Biotechnology 25: 829-833.

Krechel A., Faupel A., Hallmann J., Ulrich A., Berg G. 2002. Potato-associated bacteria and their antagonistic potential towards plant-pathogenic fungi and the plant-parasitic nematode Meloidogyne incognita (Kofoid \& White) Chitwood. Canadian Journal of Microbiology 48 (9): 772- 786.

Kubicek C.P., Mach R.L., Peterbauer C.K., Lorito M. 2001. Trichoderma: from genes to biocontrol. Journal of Plant Pathology 83 (Special Issue): 11-24.

Kurzawińska H., Gajda I. 2002. Grzyby zasiedlające sucho gnijące bulwy ziemniaka. [Fungi settling dry rotting potato tubers]. Biuletyn Instytutu Hodowli i Aklimatyzacji Roślin 223/224: 315-319.

Kurzawińska H., Klima E. 1999. Dry rot fungi occurring in potato tubers. Horticulture and Vegetable Growing 18 (3): 216-222.

Kwaśna H., Chełkowski J., Zajkowski P. 1991. Grzyby (Mycota). [Fungi (Mycota)]. Vol. XXII. Sierpik (Fusarium). Polska Akademia Nauk, Flora Polska, Warszawa, Poland, 152 pp.

Lazarovits G., Hill J., Patterson G., Conn K.L., Crump N.S. 2007. Edaphic soil levels of mineral nutrients, $\mathrm{pH}$, organic matter, and cationic exchange capacity in the geocaulosphere associated with potato common scab. Phytopathology 97 (9): 1071-1082.

Lenc L. 2006. Rhizoctonia solani and Streptomyces scabies on sprouts and tubers of potato grown in organic and integrated systems, and fungal communities in the soil habitat. Phytopathologia Polonica 42: 13-28.

Lenc L. 2009. Occurrence of Streptomyces scabies on tubers of eight potato cultivars grown in organic and integrated system. Environmental Protection and Natural Resources 40: 669676.

Lenc L., Kwaśna H., Sadowski Cz. 2012. Microbial communities in potato roots and soil in organic and integrated production systems compared by the plate culturing method. Journal of Phytopathology 160 (7-8): 337-345. 
Lenc L., Kwaśna H., Sadowski Cz. 2011. Dynamics of the root/ soil pathogens and antagonists in organic and integrated production of potato. European Journal of Plant Pathology 131: 603-620.

Mäder P., Pfiffner L., Fliessbach A., Niggli U. 1995. Biodiversity of soil biota in biodynamic, organic and conventional farming systems. p. 45-58. In: Proceedings of the 1st ENOF Workshop on Biodiversity and Land Use: The Role of Organic Farming (J. Isart, J.J. Llerena, eds.). Bonn, Germany, 8-9 December 1995. Multitext Barcelona, Spain, 155 pp.

Manici L.M., Caputo F. 2009. Fungal community diversity and soil health in intensive potato cropping systems of the east Po valley, northern Italy. Annals of Applied Biology 155 (2): 245-258.

Martinez-Beringola M.L., Salto T., Vázquez G., Larena I., Melgarejo P., De Cal A. 2013. Penicillium oxalicum reduces the number of cysts and juveniles of potato cyst nematodes. Journal of Applied Microbiology 115 (1): 199-206.

Messiha N.A.S., Bruggen A.H.C. van, Diepeningen A.D. van, Vos O.J. de, Termorshuizen A.J., Tjou-Tam-Sin N.N.A., Janse J.D. 2007. Potato brown rot incidence and severity under different management and amendment regimes in different soil types. European Journal of Plant Pathology 119 (4): 367-381.

O’Brien M.J., Rich A.E. 1979. Potato Disease. Agriculture Handbook No 474, United States Department of Agriculture, Agricultural Research Service, Washington DC, USA, 80 pp.

Overbeek L. van, Elsas J.D. van. 2008. Effects of plant genotype and growth stage on the structure of bacterial communities associated with potato (Solanum tuberosum L.). FEMS Microbiological Ecology 64 (2): 283-296.

Penton C.R., Gupta V.V.S.R., Tiedje J.M., Neate S.M., OphelKeller K., Gillings M., Harvey P., Pham A., Roget D.K. 2014. Fungal community structure in disease suppressive soils assessed by $28 \mathrm{~S}$ LSU gene sequencing. PLoS ONE 9 (4): e93893.

Pitt J.I. 1979. The genus Penicillium and its Teleomorphic States Eupenicillium and Talaromyces. Academic Press, LondonNew York-Toronto-Sydney-San Francisco, 634 pp.

Porter S. 1995. Increasing options for cover cropping in the Northeast. SARE Project Report FNE94-066. Northeast Region SARE.

Sadowski Cz., Lenc L., Korpal W., Kawalec A. 2003. Health status of tubers od potato cultivated under organic and integrated conditions. p. 682-686. In: „Obieg pierwiastków w przyrodzie" ["Circuit Elements in Nature“] (B. Gworek, J. Misiak, eds.). Instytut Ochrony Środowiska, Warszawa, 730 pp.

Sadowski Cz., Klepin J., Baturo A., Lenc L. 2002. Zdrowotność bulw i kiełków ziemniaka uprawianego w systemie ekologicznym i konwencjonalnym. [Healthiness of tubers and sprouts of potato grown in organic and conventional system]. Zeszyty Problemowe Postępów Nauk Rolniczych 489: 95-102.

Schirmbock M., Lorito M., Wang Y.L., Hayes C.K., Arisan-Atac I., Scala F., Harman G.E., Kubicek C.P. 1994. Parallel formation and synergism of hydrolytic enzymes and peptaibol antibiotics, molecular mechanisms involved in the antagonistic action of Trichoderma harzianum against phytopatho- genic fungi. Applied and Environmental Microbiology 60 (12): 4364-4370.

Seifert K., Morgan-Jones G., Gams W., Kendrick B. 2011. The Genera of Hyphomycetes. CBS-KNAW Fungal Biodiversity Centre, Utrecht, The Netherlands, 997 pp.

Shannon D., Sen A.M., Johnson D.B. 2002. A comparative study of the microbiology of soils managed under organic and conventional regimes. Soil Use and Management 18 (Suppl. 1): 274-283.

Spohn M. 2015. Microbial respiration per unit microbial biomass depends on litter layer carbon-to-nitrogen ratio. Biogeosciences 12: 817-823.

Steinberg C., Edel-Hermann V., Alabouvette C., Lemanceau P. 2007. Soil suppressiveness to plant diseases. p. 455-478. In: “Modern Soil Microbiology” (J.D. van Elsas, J. Jansson, J.T. Trevors, eds.) 2nd ed. CRC Press, Inc, Boca Raton, FL, USA, $646 \mathrm{pp}$.

Tagawa M., Tamaki H., Manome A., Koyama O., Kamagata Y, 2010. Isolation and characterization of antagonistic fungi against potato scab pathogens from potato field soils. FEMS Microbiological Letters 305 (2): 136-142.

Tischler W. 1949. Grundzüge der terrestrischen Tierökologie. Friedrich Vieweg und Sohn, Braunschweig, Germany, 220 pp. (in German)

Vinten A.J.A., Whitmore A.P., Bloem J., Howard R., Wright F. 2002. Factors affecting $\mathrm{N}$ immobilisation/mineralisation kinetics for cellulose-, glucose- and straw-amended sandy soils. Biology and Fertility of Soils 36 (3): 190-199.

Vries F.T. de, Hoffland E., Eekeren N.J.M. van, Brussaard L., Bloem J. 2006. Fungal/bacterial ratios in grasslands with contrasting nitrogen management. Soil Biology and Biochemistry 38 (8): 2092-2103.

Weinert N., Meincke R., Gottwald C., Heuer H., Gomes N.C., Schloter M., Berg G., Smalla K. 2009. Rhizosphere communities of genetically modified zeaxanthin-accumulating potato plants and their parent cultivar differ less than those of different potato cultivars. Applied and Environmental Microbiology 75 (12): 3859-3865.

Weller D.M., Raaijmakers J.M., Gardene, B.B.M., Thomashow L.S. 2002. Microbial populations responsible for specific soil suppressiveness to plant pathogens. Annual Review of Phytopathology 40: 309-348.

Wojciechowska-Kot H., Kiszczak E. 1981. Patogeniczne Fusaria $\mathrm{w}$ przechowalniach ziemniaka oraz ich rola w powstawaniu suchej zgnilizny. [Pathogenic Fusarium spp. in potato storehouses and their role in causing dry rot]. Biuletyn Instytutu Ziemniaka 26: 95-102.

Yamanaka T. 2003. The effect of $\mathrm{pH}$ on the growth of saprotrophic and ectomycorrhizal ammonia fungi in vitro. Mycologia 95 (4): 584-589.

Yao H., He Z., Wilson M.J., Campbell C.D. 2000. Microbial biomass and community structure in a sequence of soils with increasing fertility and changing land use. Microbial Ecology 40 (3): 223-237.

Yeates G.W., Bardgett R.D., Cook R., Hobbs P.J., Bowling P.J., Potter J.F. 1997. Faunal and microbial diversity in three Welsh grassland soils under conventional and organic management regimes. Journal of Applied Ecology 34 (2): 453-470. 